\title{
EVALUACIÓN DE LA PRODUCCIÓN DE BIOGÁS A PARTIR DE SUERO LÁCTEO A ESCALA DE LABORATORIO.
}

\section{EVALUATION OF BIOGAS PRODUCTION FROM WHEY AT LABORATORY SCALE.}

\author{
Sandra Lorena Blandón Navarrottt \\ Donald Samuel Zelaya Lanuza $¥ \# \neq$ \\ Juan Carlos Arranz Ramírez ${ }^{\$ \&}$ \\ Javier Alberto Zamora Calero ${ }^{* * *}$
}

RESUMEN: Esta investigación tuvo como finalidad evaluar la producción de biogás con suero lácteo. Inicialmente se caracterizaron las materias primas (estiércol y suero), para determinar en términos de composición química. Posteriormente fueron realizados experimentos, con un total de seis tratamientos, con 3 formulaciones en diferentes concentraciones de suero, realizando observación y colecta de datos por un periodo de 20 días, midiendo el nivel de producción de gas. La formulación que mejores resultados presento en cuanto a la producción de gas fue utilizada para cargar el biodigestor a escala, en el cual se produjo un volumen de $31.1 \mathrm{~L}$ de gas durante 31 días de funcionamiento. Se realizó una evaluación del tiempo de transferencia de calor entre el gas butano y el biogás producido, teniendo como resultado que el agua entra en ebullición en menor tiempo con gas butano que con biogás, lo que sugiere que el biogás es menos eficiente que el gas butano. El presupuesto de la tecnología de producción de biogás propuesta (agua + excretas) indicó que los costos de producción por litro de mezcla son de $\$ 0.36$ en el caso de suero y de $\$ 0.20$ para el agua, sin embargo los rendimientos para el biogás utilizando suero fueron de $243.16 \mathrm{~L}$ mensual y utilizando agua $74 \mathrm{~L}$ mensual, lo que demuestra que es más económico el uso de suero.

Palabras clave: eficiencia energética; energía renovable; energía limpia; residuos.

ABSTRAC: This research aims is to evaluate the production of biogas with whey, starting with the characterization of the raw materials (manure and whey) to determine their chemical composition. The following experiments, in total six, with three compositions of different concentrations of whey, were observed and the data evaluated for 20 days, measuring the average production rate of gas. The composition with the best results concerning the productions rate of gas was used to charge the bio-digester up to a scale of 31.1 I for 31 days at service. During this time, the heat transfer between the gas butane and the biogas that has been produced was determined. It showed that boiling water enters in less time with butane than biogas, suggesting that biogas is less efficient than butane. The proposed precondition for the technology of producing biogas (water + manure) indicates that the production costs per liter of the mixture add up to $\$ 0.36$ in case of whey and $\$ 0.20$ for water. Nevertheless, the output for biogas with whey amounts 243.16 I per month, whereas the

\footnotetext{
${ }^{+++}$Docente UNI RUACS, Nicaragua lore blandon@yahoo.com - Autor para la correspondencia

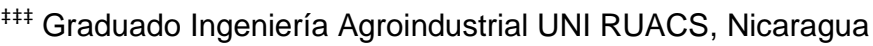

$\S \S \S$ Graduado Ingeniería Agroindustrial UNI RUACS, Nicaragua

**** Graduado Ingeniería Agroindustrial UNI RUACS, Nicaragua
} 
output with water only sums up to 74 I per months, which implies that the use of whey is much cheaper.

Keywords: efficiency, biogas, renewable energies, manure

\section{INTRODUCCIÓN}

Según (FAO, 2000) el suero es el fluido separado de la cuajada tras la coagulación de la leche, nata (crema), leche desnatada (descremada) o suero de mantequilla en la fabricación del queso, la caseína o productos similares, principalmente con enzimas del tipo cuajo. Los principales componentes de este subproducto de la industria láctea son lactosa, proteínas y sales minerales. La demanda bioquímica de oxígeno (DBO) del suero varía de 60000 a $80000 \mathrm{mg}$ de $\mathrm{O}_{2}$ por litro de agua residual (ppm), lo que depende ampliamente de la procedencia del producto (Liao \& V., 1988).

Este DBO refleja que es un líquido altamente biodegradable, por lo que la digestión anaeróbica y la generación de energías limpias es una alternativa para su aprovechamiento. En ese sentido se realizó la presente investigación, ofreciendo ventajas energéticas al producir biogás, y no consumir energía en procesos de aireación utilizados en los tratamientos tradicionales aeróbicos como los lodos activados.

\section{MATERIALES Y MÉTODOS}

Con finalidad evaluar la producción de biogás mediante la utilización de suero lácteo fueron realizados experimentos, monitoreando el volumen de gas producido y el tiempo de producción de gas.

La investigación fue desarrollada en dos etapas, en la primera etapa se llevó a cabo una caracterización de la materia prima (suero y excretas de ganado bovino) y posteriormente la determinación de la mezcla de suero con excretas que presentara el mayor rendimiento de producción de gas, para esto se utilizaron jeringas de $50 \mathrm{~mL}$ como unidades experimentales.

Los porcentajes de sólidos trabajados para las diferentes mezclas a evaluar, fueron: 10\%, $15 \%$ y $20 \%$ para cada formulación con suero y agua. Para conocer la cantidad de suero a agregar por cada kg de excreta, se utilizó la ecuación (1) propuesta por (Varnero Moreno, 2011)

$$
\% \text { ST Carga diluida }=\frac{1 \mathrm{~kg} \text { excreta fresca } * \% \text { ST Excreta fresca }}{1 \mathrm{~kg} \text { excreta fresca }+ \text { Suero } / \text { Agua agregada }}
$$

Dónde: \% ST Carga diluida: equivale a los tres porcentajes planteados $10 \%, 15 \%$ y $20 \%$ \% ST Excreta Fresca: fueron los calculados en la caracterización de materia prima.

A partir de los resultados de la primera etapa, fueron montados biodigestores (figura 1) construidos con recipientes plásticos de 25 litros, con orificio en la tapa para adaptar la tubería de $1 / 2$ pulgada y conseguir la salida del gas y poder almacenarlo en un contenedor de gas inflable. 


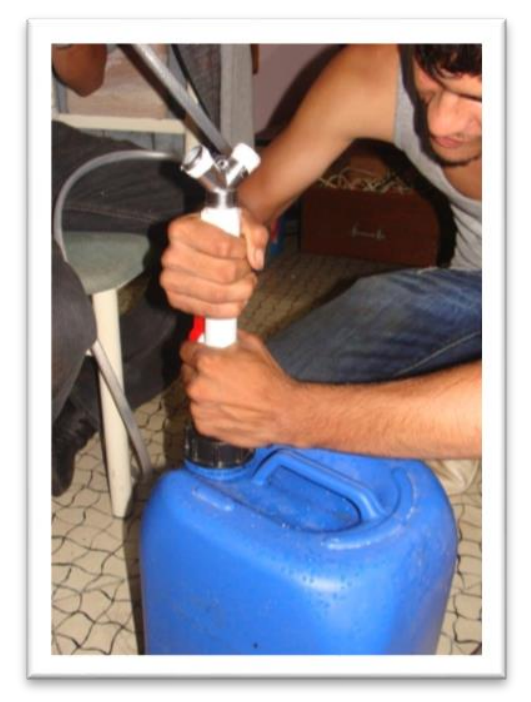

Fig. 1: Montaje de Biogidestor

El biogás producido se evaluó a través la medición del tiempo de transferencia de calor, mediante la prueba de agua hirviendo (WBT), que consiste en medir el tiempo en que se tarda determinado gas en llevar una cantidad de agua (en este caso $200 \mathrm{ml}$ ) a su punto de ebullición $\left(100^{\circ} \mathrm{C}\right)$.

Finalmente fue determinado el costo marginal y beneficio marginal haciendo un análisis presupuestario parcial del uso de biogás producido con suero en comparación al biogás producido con agua.

\section{RESULTADOS Y DISCUCIÓN}

El suero dulce presenta un $\mathrm{pH}$ de 6.12 y una densidad de $1.022 \mathrm{gr} / \mathrm{cm}^{3}$ y 6 Brix. Por su parte el suero ácido presentó un $\mathrm{pH}$ de 4.06 y la densidad de $1.022 \mathrm{gr} / \mathrm{cm}^{3}$, sin embargo el refractómetro no marcó ninguna lectura en lo referente a los grados Brix, por lo que se deduce que su valor es de cero. El resultado del cálculo de sólidos totales se resume en la Tabla 1.

Tabla 1. Porcentaje de sólidos totales de la excreta de ganado bovino.

\begin{tabular}{|ccccccc|} 
Muestra & $\begin{array}{c}\text { Peso de la } \\
\text { placa } \mathbf{( g )}\end{array}$ & $\begin{array}{c}\text { Cantidad } \\
\text { inicial de } \\
\text { excreta } \mathbf{( g )}\end{array}$ & $\begin{array}{c}\text { Peso final } \\
\text { con placa } \\
\mathbf{( g )}\end{array}$ & $\begin{array}{c}\text { Sólidos } \\
\text { totales }\end{array}$ & Humedad & $\begin{array}{c}\text { \% sólidos } \\
\text { totales }\end{array}$ \\
\hline $\mathbf{1}$ & 7.2 & 20 & 16.5 & 9.3 & 10.7 & 46.5 \\
\hline $\mathbf{2}$ & 7.1 & 20 & 16.6 & 9.5 & 10.5 & 47.5 \\
\hline $\mathbf{3}$ & 7.2 & 20 & 16.4 & 9.2 & 10.8 & 46 \\
\hline $\mathbf{4}$ & 7.1 & 20 & 16.6 & 9.5 & 10.5 & 47.5 \\
\hline $\mathbf{5}$ & 7.1 & 20 & 16.6 & 9.5 & 10.5 & 47.5 \\
\hline & & & & & Promedio & $\mathbf{4 7}$ \\
\hline
\end{tabular}


El porcentaje promedio de la excreta con que se trabajó fue de $47 \%$, cantidad que se encuentra entre los valores presentado por Varnero Moreno (2011). Con este porcentaje obtenido, se realizó el cálculo de la cantidad de suero a mezclar.

En relación a la selección de la formulación con mejor rendimiento en cuanto a la producción de gas, en la Figura 2 puede ser observado que hubo diferencia significativa entre cada uno de los tratamientos, en relación al contenido de sólidos totales y el disolvente (agua o suero). Asimismo, el promedio más alto se alcanzó con la formulación al $20 \%$ de sólidos totales y suero $(A)$, considerándose en esta investigación como la mejor formulación para la evaluación de la producción de biogás utilizando suero lácteo.

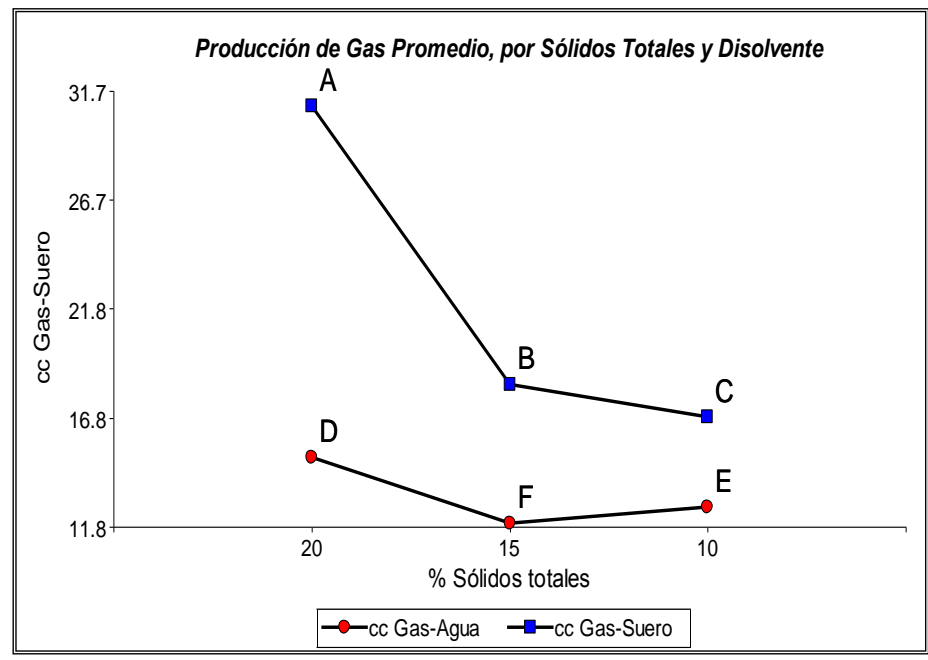

Fig. 2. Separación de las medias de la producción de gas, por sólidos totales y disolvente, Prueba de Duncan al $5 \%$.

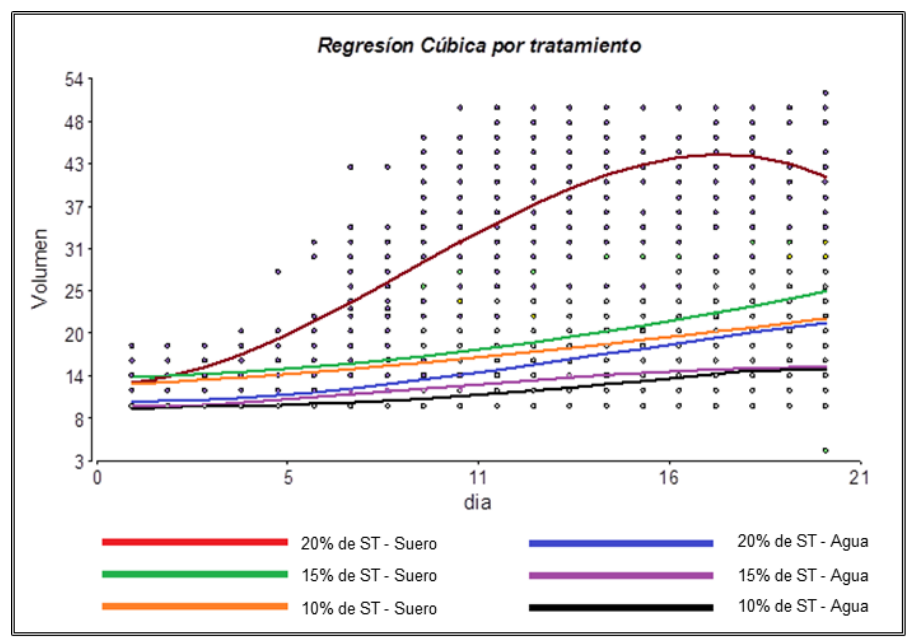

Fig. 3. Resumen de regresiones cúbicas por tratamiento

Se realizó además, una regresión no lineal cúbica para poder comparar las variables tiempo y niveles de producción de todos los tratamientos juntos, en la Figura 3 se puede observar que todos los tratamientos que utilizan suero fueron más efectivos que los tratamientos que utilizan agua como disolvente, por lo tanto el uso de suero lácteo para la producción de biogás es más eficiente ya que se logran alcanzar niveles de producción de gas más altos en menor tiempo. 
En relación a la generación de biogás, se trabajó con suero dulce, considerando el pH cercano a la neutralidad que este posee. Considerando el proceso de acidificación del suero, se hizo una segunda medición del pH a las 24 horas, tomando una muestra de 100 $\mathrm{mL}$ de la mezcla que estaba dentro de los reactores (biodigestores) y se observó el pH en ambos se encontraba en un valor de 4, por lo que se elevó el pH con óxido de calcio (cal), utilizando la relación de $1.5 \mathrm{~g}$ de cal por cada $100 \mathrm{~mL}$ de muestra.

Se hicieron 5 mediciones de pH después de puesto en funcionamiento, posteriores a la neutralización, con intervalo de tiempo de 24 horas cada una, y el valor que se obtuvo en las mediciones de $\mathrm{pH}$ fue en el rango 6 y 7.

Los resultados del calentamiento de agua con butano y biogás son presentados en las tablas 2 y 3

Tabla 2: Temperatura y tiempo de calentamiento del agua utilizando biogás como combustible

\begin{tabular}{|ccc|}
$\begin{array}{c}\text { Temperatura } \\
\left({ }^{\circ} \mathbf{C}\right)\end{array}$ & $\begin{array}{c}\text { Primera } \\
\text { prueba* }^{*}\end{array}$ & $\begin{array}{c}\text { Segunda } \\
\text { prueba* }^{*}\end{array}$ \\
\hline $\mathbf{2 6}$ & $00: 00$ & $00: 00$ \\
\hline $\mathbf{4 5}$ & $01: 49$ & $01: 51$ \\
\hline $\mathbf{6 2}$ & $02: 05$ & $02: 09$ \\
\hline $\mathbf{7 0}$ & $02: 29$ & $02: 33$ \\
\hline $\mathbf{8 2}$ & $03: 14$ & $03: 17$ \\
\hline $\mathbf{8 5}$ & $03: 28$ & $03: 31$ \\
\hline $\mathbf{8 8}$ & $03: 57$ & $04: 01$ \\
\hline $\mathbf{9 3 . 1}$ & $04: 25$ & $04: 28$ \\
\hline $\mathbf{9 5 . 9}$ & $04: 44$ & $04: 49$ \\
\hline $\mathbf{9 6 . 6}$ & $04: 56$ & $04: 59$ \\
\hline $\mathbf{1 0 0}$ & $\mathbf{0 5 : 1 2}$ & $\mathbf{0 5 : 1 5}$ \\
\hline
\end{tabular}

${ }^{*}$ Datos en minutos y segundos.
Tabla 3: Temperatura y tiempo de calentamiento del agua utilizando gas butano como combustible

\begin{tabular}{|ccc|}
\hline $\begin{array}{c}\text { Temperatura } \\
\left({ }^{\circ} \mathbf{C}\right)\end{array}$ & $\begin{array}{c}\text { Primera } \\
\text { prueba* }^{*}\end{array}$ & $\begin{array}{c}\text { Segunda } \\
\text { prueba* }\end{array}$ \\
\hline 26 & $00: 00$ & $00: 00$ \\
\hline 45 & $00: 44$ & $00: 48$ \\
\hline 62 & $01: 18$ & $01: 15$ \\
\hline 70 & $01: 31$ & $01: 33$ \\
\hline 82 & $02: 01$ & $02: 10$ \\
\hline 85 & $02: 08$ & $02: 19$ \\
\hline 88 & $02: 19$ & $02: 30$ \\
\hline 93.1 & $02: 37$ & $02: 45$ \\
\hline 95.9 & $02: 57$ & $03: 08$ \\
\hline 96.6 & $03: 05$ & $03: 17$ \\
\hline $\mathbf{1 0 0}$ & $\mathbf{0 3 : 1 8}$ & $\mathbf{0 3 : 2 7}$ \\
\hline
\end{tabular}

De los resultados presentados en las Tablas 2 y 3 puede sugerirse que el gas butano tiene menor mejor desempeño en el calentamiento de agua, ya que con este se necesitaron 3 minutos y 27 segundos para que el agua alcance $100{ }^{\circ} \mathrm{C}$, en cambio con biogás se necesitaron 5 minutos y 15 segundos para alcanzar la misma temperatura.

La comparación de la producción de biogás utilizando agua (base) y la producción de biogás utilizando suero lácteo (alternativa), se tomó en cuenta las medías de producción de los tratamientos con porcentajes de $20 \%$ con suero y con agua, obtenidos en el experimento de la determinación de la mezcla con mayor rendimiento de producción de gas.

Se estimó una carga diaria de 33.33 L/día para un biodigestor con agua con una producción de biogás $2.47 \mathrm{~L} /$ día, en el caso de un biodigestor con suero la carga diaria estimada fue de 52.63 L/día con una producción de biogás en L/día de 8.11. Con estos valores se calcularon los costos de producción diarios que fueron de U\$ 0.20 para el biodigestor con agua y de $U \$ 0.36$ para el biodigestor con suero. Se determinó también el precio por litro de 
mezcla dividendo el costo de producción entre la cantidad de mezcla por día. El resultado para la mezcla con agua es de $\$ 0.0059$ y $\$ 0.0074$ para la mezcla con suero.

El rendimiento de la producción de biogás, se calculó a partir de los promedios de producción de gas con las formulaciones de $20 \%$ de suero y las de agua. Considerando que en 20 días la producción media de gas con las formulaciones al $20 \%$ de sólidos totales de suero fue de $30.99 \mathrm{~mL}=0.03099 \mathrm{~L}$ y $14.99 \mathrm{~mL}=0.01499 \mathrm{~L}$ para las mezcla con agua.

Obteniendo como resultado que volumen de biogás que se produce por cada litro de mezcla de excreta con agua es de $0.07 \mathrm{~L}$ y en las mezclas de excreta con suero el volumen de biogás es de $0.15 \mathrm{~L}$.

Según Varnero Moreno (2011), 120000 L de biogás equivalen a 115.2 L de gas licuado. Con esto y teniendo en cuenta que el precio por litro de gas licuado distribuido por la empresa TROPIGAS cuesta \$0.59, se calculó el precio aproximado por litro de biogás. Con el resultado de todos los cálculos presentados anteriormente se procedió a realizar el presupuesto parcial por mes, que se presenta en la Tabla 4.

Tabla 4. Presupuesto parcial de la producción de biogás con suero lácteo.

\begin{tabular}{|lrrr|}
\hline \multicolumn{1}{|c}{ Concepto } & $\begin{array}{c}\text { Agua } \\
\text { (Base) }\end{array}$ & $\begin{array}{c}\text { Suero } \\
\text { (Alternativa) }\end{array}$ & \multicolumn{1}{c|}{ Cambio } \\
\hline Carga diaria, L/mes & 1000.00 & 1578.95 & 578.95 \\
\hline Precio de mezcla, \$/L & 0.0059 & 0.0074 & 0.0015 \\
\hline Costo de mezcla total \$ & 5.93 & 11.72 & 5.80 \\
\hline Rendimiento (Producción de biogás, L) & 74.00 & 243.16 & 169.16 \\
\hline Precio de biogás \$/L & 0.0422 & 0.1386 & 0.0964 \\
\hline Ingreso total & 3.12 & 33.70 & 30.58 \\
\hline Ingreso Neto & -2.81 & 21.98 & 24.79 \\
\hline Tasa de retorno marginal & & & 4.28 \\
\hline
\end{tabular}

Los costos de mezcla total se calcularon haciendo una multiplicación de la carga diaria y el precio por litro de la mezcla, puede observarse que en el caso del suero el costo de mezcla total resulta ser $\$ 5.80$ mayor que el costo de la mezcla con agua, lo que hace referencia al costo marginal, lo que indica que cambiar el uso de agua por suero en la mezcla para producir biogás, aumentaría el costo en $\$ 5.80$.

En relación al rendimiento de la producción de biogás, se nota que en el caso del suero se produce $169.2 \mathrm{~L} / \mathrm{mes}$, más de lo que se produce en un mes utilizando agua en la mezcla. Suponiendo que se vendiera el biogás se tendrían mejores ingresos utilizando suero en lugar de agua, puesto que el ingreso neto o beneficio marginal es la diferencia entre el ingreso total y el costo de mezcla total, y como resultado se obtuvo $\$ 24.79$ más utilizando suero en la mezcla por mes, esto indica que la utilización del suero provocaría un aumento 
en el ingreso o beneficio marginal nuevamente indicando que se vendiera el biogás producido.

Analizando la tasa de retorno marginal se puede observar que al utilizar suero el beneficio seria que por \$ 1 invertido se obtendrá \$ 4.28 más, lo que indica que la aplicación de esta tecnología es más rentable en relación a la tecnología base analizada( biogás producido con agua).

\section{CONCLUSIONES}

Las mezclas con $20 \%$ de sólidos totales presentaron desempeño en la producción de biogás, con un promedio de producción de $30.99 \mathrm{~mL}$ utilizando suero como disolvente, a diferencia que la mezcla con agua que produjo $14.99 \mathrm{~mL}$.

Fue posible generar biogás utilizando suero lácteo y excretas de ganado bovino, en una mezcla al $20 \%$ de sólidos totales, en biodigestores discontinuos a escala de laboratorio, en un tiempo de 19 días, sin embargo los biodigestores tuvieron un tiempo total de producción de biogás de 31 días aproximadamente.

El gas butano posee mayor poder calórico que el biogás, llevando en un menor tiempo el agua a su punto de ebullición. Esto puede atribuirse a que la presión con que se transporta el gas butano al quemador era mayor, por consiguiente la llama es más estable, en cambio la presión que se tenía con el biogás al momento de hacer la prueba no era constante, lo que provocaba variaciones en el tamaño de la flama.

La producción de biogás a partir del suero lácteo resulta ser una manera eficiente de aprovechar este residuo, debido a su efectividad en dicho proceso ya que existen muchos lugares de Nicaragua donde la generación de este residuo es alta y su aprovechamiento es mínimo.

\section{REFERENCIAS}

FAO. (2000). CODEX ALIMENTARIUS: Leche y productos lácteos. CODEK STAN A-151995, 50. FAO.

Liao, P. H., \& V., L. K. (1988). Methane Production From Cheese Whey . Vancouver.

Varnero Moreno, M. T. (2011). Manual de Biogas. Santiago de Chile: FAO.

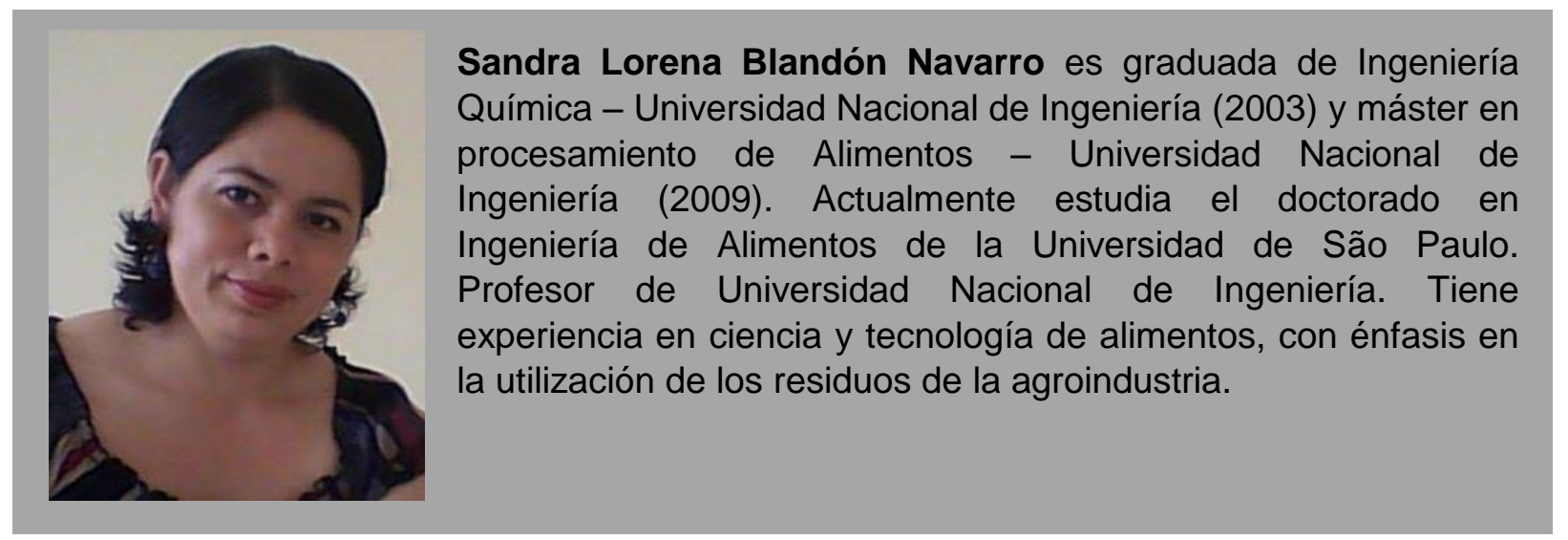

